\title{
BMJ Open Protocol for a prospective open-label clinical trial to investigate the utility of concurrent TBS/fNIRS for antidepressant treatment optimisation
}

\author{
Rebecca L D Kan, ${ }^{1}$ Arthur D P Mak (D) , 2 Sherry K W Chan, ${ }^{3}$ Bella B B Zhang (D) , ${ }^{1}$ \\ Kenneth N K Fong, ${ }^{1}$ Georg S Kranz (i) ${ }^{1,4,5}$
}

To cite: Kan RLD, Mak ADP, Chan SKW, et al. Protocol for a prospective open-label clinical trial to investigate the utility of concurrent TBS/fNIRS for antidepressant treatment optimisation. BMJ Open 2022;12:e053896. doi:10.1136/ bmjopen-2021-053896

- Prepublication history for this paper is available online. To view these files, please visit the journal online (http://dx.doi. org/10.1136/bmjopen-2021053896).

Received 27 May 2021 Accepted 15 January 2022

Check for updates

(C) Author(s) (or their employer(s)) 2022. Re-use permitted under CC BY-NC. No commercial re-use. See rights and permissions. Published by BMJ.

For numbered affiliations see end of article.

Correspondence to

Dr Georg S Kranz;

georg.kranz@polyu.edu.hk

\section{ABSTRACT}

Introduction Repetitive transcranial magnetic stimulation (rTMS) with theta burst stimulation (i.e. TBS) of the dorsolateral prefrontal cortex (DLPFC) is an innovative treatment for major depressive disorder (MDD). However, fewer than $50 \%$ of patients show sufficient response to this treatment; markers for response prediction are urgently needed. Research shows considerable individual variability in the brain responses to rTMS. However, whether differences in individual DLPFC modulation by rTMS can be used as a predictive marker for treatment response remains to be investigated. Here, we present a research programme that will exploit the combination of functional near-infrared spectroscopy (fNIRS) with brain stimulation. Concurrent TBS/fNIRS will allow us to systematically investigate TBS-induced modulation of blood oxygenation as a proxy for induced brain activity changes. The findings from this study will (1) elucidate the immediate effects of excitatory and inhibitory TBS on prefrontal activity in TBS treatment-naïve patients with MDD and (2) validate the potential utility of TBS-induced brain modulation at baseline for the prediction of antidepressant response to 4 weeks of daily TBS treatment.

Methods and analysis Open-label, parallel-group experiment consisting of two parts. In part 1, 70 patients and 37 healthy controls will be subjected to concurrent TBS/fNIRS. Intermittent TBS (iTBS) and continuous TBS (cTBS) will be applied on the left and right DLPFC, respectively. fNIRS data will be acquired before, during and several minutes after stimulation. In part 2, patients who participated in part 1 will receive a 4 week iTBS treatment of the left DLPFC, performed daily for 5 days per week. Psychometric evaluation will be performed periodically and at 1 month treatment follow-up. Statistical analysis will include a conventional, as well as a machine learning approach.

Ethics and dissemination Ethics approval was obtained from the Institutional Review Board. Findings will be disseminated through scientific journals, conferences and university courses.

Trial registration number NCT04526002.

\section{INTRODUCTION}

Stratified medicine is still an unmet need for biological psychiatry. Despite major efforts
Strengths and limitations of this study

- Concurrent application of transcranial magnetic stimulation (TMS) and functional near-infrared spectroscopy (fNIRS).

- Investigation of the immediate effects of excitatory and inhibitory theta burst stimulation (TBS) on prefrontal activity in major depression.

- Exploration of the utility of TBS-induced brain modulation at baseline for the prediction of the antidepressant response to 4 weeks of daily TBS treatment.

- Concurrent TBS/fNIRS bears technical challenges that need to be remediated.

- The NIRS probe used in our study covers only a small area underneath the coil, limiting the analysis of stimulation effects to a small region of interest.

by others and us in using neuroimaging tools to uncover diagnostic and predictive markers (e.g., Lanzenberger et $a l^{1}$ ), psychiatrists are still lacking such indicators with clinical utility. ${ }^{2}$ The urgency for developing biomarkers for psychiatric disorders such as major depressive disorder (MDD) is demonstrated by the fact that mental disorders are the leading global burden in terms of years lived with disability. ${ }^{3}$ Moreover, mental disorders are associated with economic costs that are higher than cardiovascular disorders, cancer and diabetes combined. ${ }^{4}$ In light of the high percentage of treatment refractoriness, a particular need for psychiatry is to uncover markers that predict the outcome of treatments before or at an early stage after treatment starts.

Theta burst stimulation (TBS), a special form of patterned repetitive transcranial magnetic stimulation (rTMS), has finally found its way into clinical practice for the treatment of MDD. TBS is safe, effective in depressed patients that are refractory to standard pharmacological treatments, and 
has the advantage of increased efficiency over standard rTMS. However, response rates for rTMS as well as TBS, while promising enough to offer this treatment (with only minor side effects) to patients with MDD, are still achieved in only about $50 \%$ of patients. ${ }^{5}$ Several attempts to predict antidepressant response were made in recent years but they only succeeded at a group level, whereas markers that are sufficiently accurate to guide decisions on an individual level are still absent. For example, baseline functional connectivity between subgenual anterior cingulate cortex and dorsolateral prefrontal cortex (DLPFC) has been proposed as a biomarker for the individualisation of the stimulation target to optimise treatment response. ${ }^{67}$ Yet, when functional connectivity-based target selection is implemented, response rates still do not exceed the $50 \%$ mark. ${ }^{8}$ Other attempts to predict response rates include measurements of cortical thickness ${ }^{9}$ or corticospinal excitability, ${ }^{10}$ as well as many other patient-related, illness-related and stimulation procedurerelated factors, for a review (see Fidalgo et $a l^{11}$ and $\mathrm{Kar}^{12}$ ).

Concurrent neuroimaging with TMS may be especially fruitful to probe diagnostic and predictive neuroimaging markers as it aims to uncover the immediate modulatory effects of stimulation. Indeed, the prevailing view on therapeutic brain stimulation is that modulation of prefrontal excitability mediates its antidepressant effect. Hence, direct modulatory effects of prefrontal excitability during and immediately after rTMS likely forecast long-lasting changes in cortical excitability by promoting synaptic plasticity, which, according to current theory, should accompany rTMS treatment response. ${ }^{10}$ Technological advances within the last decade allowed for the application of concurrent brain measurements with TMS using functional magnetic resonance imaging (fMRI) or electroencephalogram (EEG) ${ }^{13}$ Authors observed prefrontal activation on $1 \mathrm{~Hz}$ rTMS with BOLD responses correlating with increasing stimulation strength. Activations were observed during the $10 \mathrm{~s}$ stimulation blocks and lasting 4-6s after the last stimulation. ${ }^{14}$ However, although highly promising for future research, it is questionable whether such a sophisticated combination of TMS and fMRI will eventually translate into a routinely used clinical test. Moreover, simultaneous image acquisitions during the application of a stimulation-burst or train of high frequency (HF) rTMS is impossible in an fMRI setting.

Functional near-infrared spectroscopy (fNIRS) is cheap, easy and harmless to apply and a widely available method to measure superficial brain activity and connectivity by means of changes in blood haemoglobin concentrations. Concurrent TMS/fNIRS may be clinically superior to TMS/fMRI in order to probe the direct modulatory effect of prefrontal excitability during and immediately after stimulation. Indeed, a recent study from Boston University attempted to predict the antidepressant response to rTMS by using fNIRS (NCT01192685). Unfortunately, the study had to be terminated due to a technical failure of the neuronavigation system. However, more research is needed along this line because there is evidence indicating that cortical activity modulations before treatment commencement may be able to predict antidepressant treatment response. In a recent study published by Oliveira-Maia and colleagues, they measured stimulationinduced changes in motor evoked potentials (MEP) at baseline and found that modulations of corticospinal excitability predicted the antidepressant response of a 2 week daily HF rTMS treatment. ${ }^{10}$ The results of this study indicated that immediate modulatory effects of rTMS of the motor cortex forecast synaptic plasticity and associated antidepressant treatment effects. However, the motor cortex is not considered a key brain area in the neuropathology of MDD and predictions based on modulations of MEPs have been only moderately successful. ${ }^{10}$ Therefore, research is needed that probes cortical modulations directly in the DLPFC; that is, the site where therapeutic brain stimulation is applied.

Noteworthy, studies measuring cortical and corticospinal activity reveal a substantial degree of individual variability in TMS-induced modulations. ${ }^{10}{ }^{15-18}$ For example, Maeda $e t a l^{15}$ investigated the effects of $1 \mathrm{~Hz}$, $10 \mathrm{~Hz}, 15 \mathrm{~Hz}$ and $20 \mathrm{~Hz}$ rTMS on MEP shortly after stimulation. Although responses were on average lowest at $1 \mathrm{~Hz}$ and highest at $20 \mathrm{~Hz}$, authors detected a high degree of variability, with some individuals even showing the opposite pattern, that is, stronger excitatory responses at $1 \mathrm{~Hz}$ compared with $20 \mathrm{~Hz}$. A high degree of variation in cortical excitability was also reported in Oliveira-Maia et $a l^{10}$ and in a recent TMS/fMRI study. ${ }^{18}$ The aim of the current proposal is to use such individual patterns of DLPFC modulations for personalised medicine in MDD.

There are two objectives in our proposal: the first objective is to provide mechanistic evidence for the direct effects of TBS of the healthy and presumed neuropathological prefrontal cortex (PFC). Specifically, we aim to assess excitability modulations of prefrontal oxyhaemoglobin $(\mathrm{HbO})$ by applying TBS on DLPFC in patients with MDD compared with healthy controls (HC). The second objective is to evaluate the relationship between immediate excitability modulations of the DLPFC and treatment response and thus provide a novel biomarker for individual patient selection. Given previous evidence and based on our line of reasoning given above, we will test the following operational hypotheses regarding our first objective: (1) We hypothesise an average (overall participants) increase in prefrontal $\mathrm{HbO}$ on intermittent TBS (iTBS) and an average decrease in $\mathrm{HbO}$ on continuous TBS (cTBS) in MDD and HC. ${ }^{19} 20$ (2) We hypothesise average changes in prefrontal $\mathrm{HbO}$ will occur during stimulation, compared with baseline. ${ }^{19} 21$ (3) We hypothesise significantly increased variance in $\mathrm{HbO}$ responses during and after stimulation compared with baseline. ${ }^{10-18}$ (4) We hypothesise that patients with MDD have lower variability in TBS-induced $\mathrm{HbO}$ modulations compared with HC. ${ }^{1022} 23$ The following operational hypothesis will be tested regarding our second objective: (5) we hypothesise that individual TBS-induced $\mathrm{HbO}$ 


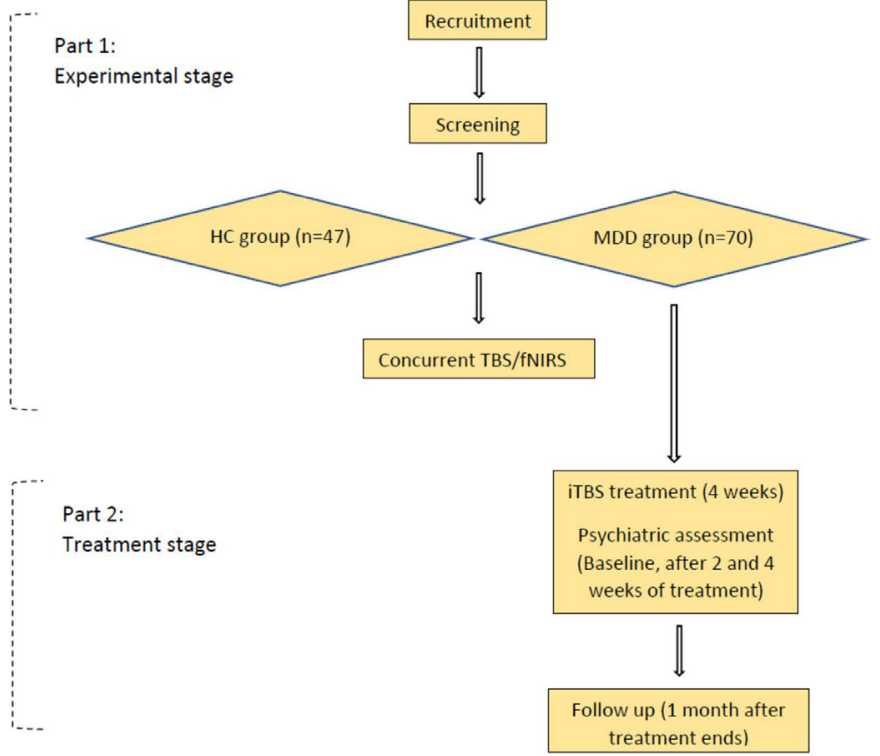

Figure 1 Study design. HC, healthy controls; fNIRS, functional near-infrared spectroscopy; MDD, major depressive disorder; iTBS, intermittent TBS; TBS, theta burst stimulation.

modulations predict the antidepressant response after treatment. $^{22} 23$

\section{METHODS AND ANALYSIS \\ Study design}

The study is designed as an open-label, parallel-group experiment and has two parts (see figure 1). In part 1, patients with MDD and $\mathrm{HC}$ will be subjected to concurrent TBS/fNIRS. iTBS will be applied on the left DLPFC, whereas cTBS will be applied on the right DLPFC. Each participant will receive iTBS first, followed by cTBS after a delay of 1 hour to exclude possible interaction effects. ${ }^{24}$ fNIRS data acquisition will include a baseline measurement of a few minutes, will continue during the stimulation period and last for several minutes poststimulation period. The length of the poststimulation period will be optimised to cover the entire duration of anticipated facilitatory effects of iTBS. ${ }^{24}$ The TMS operator, as well as the researchers performing data analyses will be blinded regarding group membership. In part 2, patients who participated in part 1 will receive a 4 -week brain stimulation treatment trial with iTBS of the left DLPFC, performed daily for 5 days per week. Patients and doctors prescribing and evaluating the treatment as well as TMS operators administering the treatment are kept blind to fNIRS results. Psychometric evaluation will be performed on the day of TBS/fNIRS measurements and at the day of treatment start, as well as after 2 and after 4 weeks of treatment. A follow-up assessment will be performed 1 month after treatment ends.

\section{Participants}

Seventy patients diagnosed with MDD in a current major depressive episode will be included. Key inclusion criteria are: MDD (DSM-5), 18 $\leq$ age $\leq 60$, Hamilton depression rating scale (HAMD-17) $\geq 18$, approval for TBS treatment by the physician in charge, stable antidepressive medication 4 weeks before treatment. Key exclusion criteria are: a history of brain surgery, head injury, stroke or neurodegenerative disorder, diagnosis of personality disorder, psychotic features, active suicidal intent, severe somatic comorbidities, cardiac pacemakers, deep brain stimulation, intracranial metallic particles, history of seizures, antiepileptics and benzodiazepines corresponding to a dose of $>1 \mathrm{mg}$ lorazepam/day, substance dependence or abuse, if it is the primary clinical problem. For the HC group, key inclusion criteria are: age between 18 and 60, right-handedness. Key exclusion criteria are: a current or previous diagnosis of a psychiatric, neurological disorder or severe internal illness, common contraindications to rTMS, ${ }^{25}$ and a psychiatric disorder in their first-degree relatives.

The sample size was determined based on previous studies demonstrating that motor cortex excitability modulation significantly predicts antidepressant response of a 2 week rTMS treatment. ${ }^{10}$ A minimum sample size of 37 was determined using an effect size of $\mathrm{r}=0.43,{ }^{10} \mathrm{a}$ power of 0.8 and an alpha of 0.05 using $G^{*}$ Power 3.0.10 (Point biseral correlation model, two-tailed). Given an expected dropout rate of $15 \%$, conservative sample size was set to 43 participants. However, in order to ensure adequate power and the ability to have reliable estimates and replicable findings, we aim to include a sample size of 70 or above. In addition, we will recruit $47 \mathrm{HC}$ to participate in the TBS/fNIRS measurement. The sample is based on the comparison of MEP facilitation obtained in MDD $(8 \pm 49 \%)^{10}$ and HC $(37.9 \pm 53.6 \%),{ }^{15}$ determined using an effect size of $\mathrm{d}=0.58$, a power of 0.8 and an alpha of 0.05 using $G^{*}$ Power V.3.0.10 (unpaired two sample t-test, two-tailed). Four sites will be involved in patient recruitment: (1) the Department of Psychiatry, Chinese University of Hong Kong and its associated hospital, the Prince of Wales Hospital (Dr Arthur Mak, Co-I); (2) the OT outpatient clinic at the Department of Rehabilitation Sciences, The Hong Kong Polytechnic University (Dr Kenneth Fong, Co-I); (3) the Department of Psychiatry, Kowloon hospital (Dr Wai Ching Yan, Dr Athena K Y Chan) and (4) the Department of Psychiatry, The University of Hong Kong and its associated hospital, the Queen Mary Hospital (Dr Sherry K W Chan, Co-I). HC participants will be recruited via posters and leaflets displayed at billboards on the university campus and community centres, community websites and social media. Participants will be screened by an experienced psychiatrist and the study including all study-related procedures will be explained to participants in oral and written form prior inclusion. The study will be performed in accordance with the Declaration of Helsinki ${ }^{26}$ including current revisions. All participants will be asked for written informed consent prior to inclusion in the study. Participants can decide to withdraw from the study at any time. The investigator may remove participants from the trial if exclusion 
criteria have been met or ending the participation is in the interest of the participant or study.

\section{Theta-burst stimulation}

TBS comprises 3-pulse $50 \mathrm{~Hz}$ bursts, applied every 200 ms (at $5 \mathrm{~Hz})$ as described previously. ${ }^{24}$ iTBS consists of $2 \mathrm{~s}$ trains with an inter-train-interval of $8 \mathrm{~s}$. We will repeat trains (30 pulses; 10 bursts) for 20 times to reach a total number of 600 pulses $(3 \times 10 \times 20)$. cTBS will comprise uninterrupted bursts to reach a total number of 600 pulses. ${ }^{27}$ Concurrent TBS/fNIRS stimulation will be applied over the left (iTBS) and right (cTBS) DLPFC at an intensity of $90 \%$ resting motor threshold (RMT), this corresponds to $\sim 110 \%$ of the active motor threshold, an intensity that also elicited robust DLPFC activation in a recent concurrent TMS/fMRI study. ${ }^{14}$ Stimulation at $90 \%$ RMT will also ensure compliance, reduce sensory discomfort and minimise dropout rates during the concurrent TBS/ fNIRS experiment. Still, scalp discomfort will be recorded directly after the stimulation. We refrained from choosing an intensity of $120 \%$ RMT (which will be applied during antidepressant treatment) for the concurrent TBS/fNIRS experiment because such intensity would unlikely be tolerated by all patients as they are stimulation-naïve at the time of the experiment. The stimulation site over the DLPFC will be determined using the international 10-20 system and corresponds to the F3 label, determined using the optimised method by Beam et al. ${ }^{28}$ TBS of the PFC is generally well tolerated, even at higher stimulation intensities. Antidepressant treatment comprises daily sessions of iTBS of the left DLPFC, five times a week for 4 weeks. Stimulation intensity will be $120 \%$ RMT (titration to full therapeutic dose over the first 3 days), as approved by the Food and Drug Administration in the USA. ${ }^{5}$ The stimulation site will be the same as in the concurrent TBS/ fNIRS stimulation. Treatment will be performed at the TMS treatment centres of the participating local clinics (Department of Psychiatry, The University of Hong Kong and The Chinese University of Hong Kong and at the Department of Rehabilitation Sciences, The Hong Kong Polytechnic University).

\section{Functional near-infrared spectroscopy}

We will make use of the fNIRS system Imagent from ISS, Champaign, Illinois, USA (http://www.iss.com/biomedical/instruments/imagent.html) to determine changes in haemoglobin concentrations before, during and after TBS. Imagent uses a sensor that is embedded in a rectangular rubber pad with prisms inside so that optical fibres are rested tangentially instead of perpendicularly on the head surface. This arrangement allows the placement of the sensor directly underneath the TMS coil in close proximity to the stimulation site. Concurrent TBS/fNIRS poses technical and conceptual challenges. Although NIRS has the advantage of being relatively insensitive to motion artefacts, pilot data from our lab show significant stimulationrelated artefacts if sensors and the TMS coil touch each other. Hence, a minimum distance of a few millimetres between probes and the TMS coil will be ensured before the start of measurements. The lowest fNIRS probes will match the Fp1-Fp2 line in order to cover most of the PFC extending to the temporal lobes (DLPFC, ventrolateral, frontopolar and superior temporal regions). The device measures changes in $\mathrm{HbO}$, deoxy-haemoglobin (HbR) and total haemoglobin ( $\mathrm{HbT})$ using two wavelengths of infrared light $(695$ and $830 \mathrm{~nm})$. With a source-detector spacing of $3 \mathrm{~cm}$, changes in $\mathrm{Hb}$ can be measured at a depth of $2-3 \mathrm{~cm}$ corresponding to the cerebral cortex. fNIRS is not a perfect measure of brain activity and there are several sources of artefacts that need to be carefully considered. We will primarily focus on $\mathrm{HbO}$ since it may closer reflect BOLD changes as measured by fMRI. ${ }^{29}$ The primary imaging endpoint will be the mean $\mathrm{HbO}$ amplitude of left and right DLPFC during and after the TBS stimulation. Secondary endpoints include mean $\mathrm{Hb}$ amplitudes during and after iTBS and cTBS, the steepness in the decline of $\mathrm{Hb}$ and ascent of $\mathrm{HbO}$ values, as well as the area under curve of $\mathrm{HbO}$ and $\mathrm{Hb}$ values during stimulation. During concurrent TBS/fNIRS, participants will comfortably sit in a chair. Participants will be carefully instructed about the nature of the experiment prior to the TBS/fNIRS run.

\section{Psychiatric assessment}

HAMD17 is a standard instrument used in most clinical trials to screen for MDD. We will use a baseline score of HAMD17 $\geq 18$ to apply a generally accepted definition of depression severity as inclusion criteria and to ensure comparability with other clinical trials. However, we will use the Montgomery-Asberg depression rating scale (MADRS) as the primary outcome measure because this symptom rating scale is more sensitive to changes over time. ${ }^{30} 31$ In addition, we will also use the Patient Health Questionnaire 9 (PHQ-9) as self-report questionnaire to assess subjective treatment effects over time. The PHQ-9 is widely used in psychiatric research. Therefore, we use different instruments for inclusion criteria and measurement of treatment response including both rater assessment and self-report inventories, a common practice in many clinical trials.

All psychometric scales used in this study are available in Chinese, ${ }^{32-34}$ and show comparable psychometric qualities compared with the original scales. For example, the inter-rater reliability of the Chinese version of HAMD was $\mathrm{r}=0.94$, as was the sensitivity $(0.79)$ and specifictity $(0.80) .{ }^{32}$ The Chinese version of MADRS and Quick inventory of depressive symptomatology-clinician rating (QIDS-C) shows a high correlation with the HAMD (0.853 and 0.75 , respectively). ${ }^{33}$ The Chinese version of all scales have been used in several previous clinical trials which involved Hong Kong populations. ${ }^{35} 36$

Psychiatric assessment includes a range of clinical scales, administered at baseline on the day of the TBS/fNIRS measurement, after 2 and 4 weeks of treatment, as well as at follow-up 1 month after treatment ends. The primary clinical outcome measure will be the response rate after 
treatment (defined by a Chinese-MADRS (C-MADRS) score reduction $\geq 50 \%$ of baseline). Secondary endpoints will be the remission rate after treatment (defined by a C-MADRS $\leq 7$ ), cut-off scores for the C-MADRS are based on Liu et al. ${ }^{33}$ Further, secondary endpoints include the absolute reduction of mean Chinese-HAMD17 (C-HAMD17) and Chinese-IDS-C (C-IDS-C) after 2 and 4 weeks of treatment and at 1-month follow-up, as well as the response (C-IDS-C and C-HAMD17 $\geq 50 \%$ of baseline) and remission rates (C-IDS-C $\leq 12$, C-HAMD17 $\leq 7$ ) of patients after 4 weeks of treatment, ${ }^{3738}$ a reduction of $50 \%$ on a depression symptom rating scale is the most common response criterium in depression trials. Adverse events (AE) will be assessed according to good clinical practice (GCP) using an AE-questionnaire to detect unwanted side effects related to the treatment. Suicidality will be evaluated on each treatment day. Similarly, depression severity will be evaluated at each treatment day using the Chinese version of the PHQ-9 (C-PHQ-9). Patients will be discontinued if they experience worsening in depression, defined as an increase in C-PHQ-9 from baseline of more than $25 \%$ during two consecutive assessments, or development of active suicidal intent or attempted suicide. Potentially occurring serious AEs will be recorded.

\section{Data processing and statistical analysis}

fNIRS data analysis will follow the standard processing steps. This includes spatial registration (recording of standard cranial landmarks nasion, inion, left and right ear and the 3D locations of the fNIRS probes); transformation to MNI space; band-pass filtering for motion artefact removal; and estimation of the haemodynamic response function using GLM, as implemented in the NIRS Toolbox for MATLAB. Comparisons between $\mathrm{HbO}$ values at baseline and during/after stimulation will reveal TBS-related de/activations. A t-test between prestimulation and poststimulation will be performed to test hypothesis 1 . Hypothesis 1 will be supported if there is a significant increase and decrease in prefrontal $\mathrm{HbO}$ after iTBS and cTBS, respectively. A t-test between prestimulation and during stimulation will be performed to test hypothesis 2. Hypothesis 2 will be supported if there is a significant change in prefrontal $\mathrm{HbO}$ during stimulation. An F-test for the comparison of the variance in $\mathrm{HbO}$ values before vs after stimulation will be performed to test hypothesis 3 . Hypothesis 3 will be supported if the F-test is significant. Similarly, an F-test for the comparison of the variance in $\mathrm{HbO}$ values in MDD vs $\mathrm{HC}$ will be performed to test hypothesis 4 . Hypothesis 4 will be supported if the F-test is significant. Analyses will be performed using the IBM SPSS software (http://www-01. ibm.com/software/analytics/spss/). The alpha level will be set at 0.05 , adjusted for multiple comparisons using the Bonferroni-Holm procedure. For hypothesis 5 , we will pursue two predictive modelling approaches, a conventional statistical analysis approach, as well as a machine learning approach. ${ }^{39}$ First, a logistic regression analysis will be performed to define significant predictors of treatment response (defined as MADRS reduction $\geq 50 \%$ of baseline, see above). Logistic regression will be calculated as implemented in the generalised linear model function 'glm' of the statistical software 'R' (https:/ / www.r-project. $\mathrm{org} /$ ). Predictors will include imaging endpoints as given above, as well as sociodemographic and psychosocial variables (including the classification of patients as pharmacologically treatment resistant, TRD, defined by a failed treatment response after two or more consecutive antidepressants of adequate duration and dosage). Hierarchical multiple linear regression models will also be calculated to determine the relationship between MADRS reductions and secondary imaging endpoints as potential response predictors. Second, we will use machine learning algorithms for the classification of patients. We will test different algorithms since there is no established rule for the choice of an optimal machine learning approach. We will start with a dichotomous classification using the RandomForest (RF) package for R (ran. r-project.org/) and determine the most useful predictors for distinguishing responders from non-responders. RF is an ensemble tree classification tool that randomly selects subsamples of observations and builds a decision tree for the optimal splitting of these observations according to an outcome variable by a combination of predictors. For each split, the best performing predictor out of a random selection is applied. RF has the advantage of being straightforward and less susceptible to overfitting compared with other machine learning classifiers. To measure the predictive power of our classification model, we will use a fivefold cross-validation design. This allows for optimal validation in the absence of an independent test set. ${ }^{40}$ There is no established method of power calculation for RF. However, we will restrict $\mathrm{HbO}$ measurements to few prefrontal channels in order to keep the number of features for classification below the number of observations, thereby preventing the problem of hyperdimensionality. Receiver operating characteristics will be plotted using the ROCR package for the R-software.

\section{Patient and public involvement}

Patients and/or the public were not involved in the design, or conduct, or reporting, or dissemination plans of this research.

\section{Ethics and dissemination}

Ethics approval was obtained from the Institutional Review Board of the Hong Kong Polytechnic University (reference numbers HSEARS20200120005, CRESC202009), as well as from the Institutional Review Boards of participating hospitals. An information sheet will be provided to participants before the experiment and a consent form will be signed by both PI and participant to protect the right of both parties. Participants will receive reimbursement for their participation. The data will be stored in an encrypted way and the accessibility is restricted to the 
researcher team. The study will start in January 2022 and is expected to be completed in December 2023.

The findings of this study will be disseminated through scientific journals, academic conferences, and university courses.

\section{Challenges and potential limitations of this study}

Although fNIRS has the advantage of being relatively insensitive to motion artefacts, we expect stimulationrelated artefacts caused by muscle contractions on the scalp given that sensors are in close proximity to the TMS coil. Furthermore, stimulation may have direct effects on superficial microvasculature. A challenge of this study will therefore be to minimise such artefacts. A variety of techniques have been proposed to resolve these and other issues related to TMS-fNIRS integration. ${ }^{41}$ Moreover, using NIRS in this study will limit the interpretation of our results due to the inherent limitations of the technique of fNIRS. This includes a restriction to measurements of shallow cortical regions (compared with fMRI) and restrictions in temporal resolution (as compared with EEG). Finally, the NIRS probe proposed to be used in our study only covers a small cortical area underneath the coil, which limits the analysis of stimulation effects to a small region of interest.

\section{Author affiliations}

${ }^{1}$ Department of Rehabilitation Sciences, The Hong Kong Polytechnic University, Hong Kong, China

${ }^{2}$ Department of Psychiatry, The Chinese University of Hong Kong, Hong Kong, China ${ }^{3}$ Department of Psychiatry, Queen Mary Hospital, The University of Hong Kong, Hong Kong, China

${ }^{4}$ Department of Psychiatry and Psychotherapy, Medical University of Vienna, Wien, Austria

${ }^{5}$ The State Key Laboratory of Brain and Cognitive Sciences, The University of Hong Kong, Hong Kong, People's Republic of China

\section{Twitter Arthur D P Mak @arthurdpmak}

Contributors GSK conceived the study and analysis. GSK and RLDK drafted the protocol and will conduct the data analysis. RLDK, BBBZ and KNKF will collect the data. ADPM and SKWC will assist in recruiting participants and performing the stimulation treatment. All the authors reviewed the protocol and agree to the final version being submitted.

Funding This work is supported by the General Research Fund (GRF) under the University Grants Committee (UGC) of the Hong Kong Special Administrative Region, grant number 15100120 .

Competing interests None declared.

Patient and public involvement Patients and/or the public were not involved in the design, or conduct, or reporting, or dissemination plans of this research.

Patient consent for publication Not applicable.

Provenance and peer review Not commissioned; externally peer reviewed.

Open access This is an open access article distributed in accordance with the Creative Commons Attribution Non Commercial (CC BY-NC 4.0) license, which permits others to distribute, remix, adapt, build upon this work non-commercially, and license their derivative works on different terms, provided the original work is properly cited, appropriate credit is given, any changes made indicated, and the use is non-commercial. See: http://creativecommons.org/licenses/by-nc/4.0/.

\section{ORCID iDs}

Arthur D P Mak http://orcid.org/0000-0003-1627-6434

Bella B B Zhang http://orcid.org/0000-0003-2580-3371

Georg S Kranz http://orcid.org/0000-0002-3892-1804
REFERENCES

1 Lanzenberger R, Kranz GS, Haeusler D, et al. Prediction of SSRI treatment response in major depression based on serotonin transporter interplay between median raphe nucleus and projection areas. Neuroimage 2012;63:874-81.

2 Kranz GS, Zhang JJQ. Expanding the collection of neuroimaging tools in psychiatry. World J Biol Psychiatry 2018;19:482-3.

3 Vigo D, Thornicroft G, Atun R. Estimating the true global burden of mental illness. Lancet Psychiatry 2016;3:171-8.

4 Smith K. Trillion-dollar brain drain. Nature 2011;478:15.

5 Blumberger DM, Vila-Rodriguez F, Thorpe KE, et al. Effectiveness of theta burst versus high-frequency repetitive transcranial magnetic stimulation in patients with depression (THREE-D): a randomised non-inferiority trial. Lancet 2018;391:1683-92.

6 Weigand A, Horn A, Caballero R, et al. Prospective validation that Subgenual connectivity predicts antidepressant efficacy of transcranial magnetic stimulation sites. Biol Psychiatry 2018;84:28-37.

7 Fox MD, Buckner RL, White MP, et al. Efficacy of transcranial magnetic stimulation targets for depression is related to intrinsic functional connectivity with the subgenual cingulate. Biol Psychiatry 2012;72:595-603.

8 Taylor SF, Ho SS, Abagis T, et al. Changes in brain connectivity during a sham-controlled, transcranial magnetic stimulation trial for depression. J Affect Disord 2018;232:143-51.

9 Boes AD, Uitermarkt BD, Albazron FM, et al. Rostral anterior cingulate cortex is a structural correlate of repetitive TMS treatment response in depression. Brain Stimul 2018;11:575-81.

10 Oliveira-Maia AJ, Press D, Pascual-Leone A. Modulation of motor cortex excitability predicts antidepressant response to prefrontal cortex repetitive transcranial magnetic stimulation. Brain Stimul 2017;10:787-94.

11 Fidalgo TM, Morales-Quezada JL, Muzy GSC, et al. Biological markers in noninvasive brain stimulation trials in major depressive disorder: a systematic review. J ECT 2014;30:47-61.

12 Kar SK. Predictors of response to repetitive transcranial magnetic stimulation in depression: a review of recent updates. Clin Psychopharmacol Neurosci 2019;17:25-33.

13 Seewoo BJ, Etherington SJ, Feindel KW, et al. Combined rTMS/fMRI studies: an overlooked resource in animal models. Front Neurosci 2018;12:180.

14 Navarro de Lara LI, Tik M, Woletz M, et al. High-sensitivity TMS/fMR of the human motor cortex using a dedicated multichannel MR coil. Neuroimage 2017;150:262-9.

15 Maeda F, Keenan JP, Tormos JM, et al. Interindividual variability of the modulatory effects of repetitive transcranial magnetic stimulation on cortical excitability. Exp Brain Res 2000;133:425-30.

16 Goldsworthy MR, Müller-Dahlhaus F, Ridding MC, et al. Inter-subject variability of LTD-like plasticity in human motor cortex: a matter of preceding motor activation. Brain Stimul 2014;7:864-70.

17 Jannati A, Block G, Oberman LM, et al. Interindividual variability in response to continuous theta-burst stimulation in healthy adults. Clin Neurophysiol 2017;128:2268-78.

18 Vink JJT, Mandija S, Petrov PI, et al. A novel concurrent TMS-fMRI method to reveal propagation patterns of prefrontal magnetic brain stimulation. Hum Brain Mapp 2018;39:4580-92.

19 Cao TT, Thomson RH, Bailey NW, et al. A near infra-red study of blood oxygenation changes resulting from high and low frequency repetitive transcranial magnetic stimulation. Brain Stimul 2013;6:922-4.

20 Tupak SV, Dresler T, Badewien M, et al. Inhibitory transcranial magnetic theta burst stimulation attenuates prefrontal cortex oxygenation. Hum Brain Mapp 2013;34:150-7.

21 Li R, Potter T, Wang J, et al. Cortical hemodynamic response and connectivity modulated by sub-threshold high-frequency repetitive transcranial magnetic stimulation. Front Hum Neurosci 2019;13:90.

22 Branchi I. The double edged sword of neural plasticity: increasing serotonin levels leads to both greater vulnerability to depression and improved capacity to recover. Psychoneuroendocrinology 2011;36:339-51.

23 Bessa JM, Ferreira D, Melo I, et al. The mood-improving actions of antidepressants do not depend on neurogenesis but are associated with neuronal remodeling. Mol Psychiatry 2009;14:764-73. 39.

24 Huang Y-Z, Edwards MJ, Rounis E, et al. Theta burst stimulation of the human motor cortex. Neuron 2005;45:201-6.

25 Rossi S, Hallett M, Rossini PM, et al. Safety, ethical considerations, and application guidelines for the use of transcranial magnetic stimulation in clinical practice and research. Clin Neurophysiol 2009;120:2008-39.

26 RICKHAM PP. Human experimentation. code of ethics of the world Medical association. Declaration of Helsinki. Br Med J 1964;2:177. 
27 Kranz G, Shamim EA, Lin PT, et al. Blepharospasm and the modulation of cortical excitability in primary and secondary motor areas. Neurology 2009;73:2031-6.

28 Beam W, Borckardt JJ, Reeves ST, et al. An efficient and accurate new method for locating the F3 position for prefrontal TMS applications. Brain Stimul 2009;2:50-4.

29 Strangman G, Culver JP, Thompson JH, et al. A quantitative comparison of simultaneous BOLD fMRI and NIRS recordings during functional brain activation. Neuroimage 2002;17:719-31.

30 Montgomery SA, Asberg M. A new depression scale designed to be sensitive to change. Br J Psychiatry 1979;134:382-9.

31 Plewnia C, Brendel B, Schwippel T, et al. Treatment of major depressive disorder with bilateral theta burst stimulation: study protocol for a randomized, double-blind, placebo-controlled multicenter trial (TBS-D). Eur Arch Psychiatry Clin Neurosci 2021;271:1231-43

32 Leung CM, Wing YK, Kwong PK, et al. Validation of the ChineseCantonese version of the hospital anxiety and depression scale and comparison with the Hamilton rating scale of depression. Acta Psychiatr Scand 1999;100:456-61.

33 Liu J, Xiang Y-T, Lei H, et al. Guidance on the conversion of the Chinese versions of the quick inventory of depressive Symptomatology-Self-Report (C-QIDS-SR) and the MontgomeryAsberg scale (C-MADRS) in Chinese patients with major depression. $J$ Affect Disord 2014;152-154:530-3.

34 Liu J, Xiang Y-T, Wang G, et al. Psychometric properties of the Chinese versions of the quick inventory of depressive symptomatology - clinician rating (C-QIDS-C) and self-report (CQIDS-SR). J Affect Disord 2013;147:421-4.

35 Ho CWH, Chan SC, Wong JS, et al. Effect of aerobic exercise training on Chinese population with mild to moderate depression in Hong Kong. Rehabil Res Pract 2014;2014:627376.

36 Shih VWY, Chan W-C, Tai O-K, et al. Mindfulness-based cognitive therapy for late-life depression: a randomised controlled trial. East Asian Archives of Psychiatry 2021;31:27-35.

37 Trivedi MH, Rush AJ, Ibrahim HM, et al. The inventory of depressive symptomatology, clinician rating (IDS-C) and self-report (IDS-SR), and the quick inventory of depressive symptomatology, clinician rating (QIDS-C) and self-report (QIDS-SR) in public sector patients with mood disorders: a psychometric evaluation. Psychol Med 2004;34:73-82.

38 Lee C-P, Liu C-Y, Hung C-I. Psychometric evaluation of a 6-item Chinese version of the Hamilton depression rating scale: Mokken scaling and item analysis. Asia Pac Psychiatry 2017;9:e12287.

39 Scheinost D, Noble S, Horien C, et al. Ten simple rules for predictive modeling of individual differences in neuroimaging. Neuroimage 2019;193:35-45.

40 Varoquaux G, Raamana PR, Engemann DA, et al. Assessing and tuning brain decoders: cross-validation, caveats, and guidelines. Neuroimage 2017;145(Pt B):166-79.

41 Parks NA. Concurrent application of TMS and near-infrared optical imaging: methodological considerations and potential artifacts. Front Hum Neurosci 2013;7:592. 